Revue de droit comparé du travail et de la sécurité sociale

3 | 2017

Le travail dans l'économie informelle, un défi pour le droit social

\title{
La constitutionnalité de la réforme de la sécurité sociale
}

Yaroslava Genova

\section{OpenEdition}

Journals

Édition électronique

URL : https://journals.openedition.org/rdctss/436

DOI : $10.4000 /$ rdctss.436

ISSN : 2262-9815

Éditeur

Centre de droit comparé du travail et de la sécurité sociale

Édition imprimée

Date de publication : 1 septembre 2017

Pagination : 192-196

ISSN : 2117-4350

Référence électronique

Yaroslava Genova, "La constitutionnalité de la réforme de la sécurité sociale », Revue de droit comparé du travail et de la sécurité sociale [En ligne], 3 | 2017, mis en ligne le 01 novembre 2017, consulté le 04 décembre 2021. URL : http://journals.openedition.org/rdctss/436 ; DOI : https://doi.org/10.4000/ rdctss. 436

\section{(c) (i) $\ominus$}

Revue de droit comparé du travail et de la sécurité sociale est mise à disposition selon les termes de la Licence Creative Commons Attribution - Pas d'Utilisation Commerciale - Pas de Modification 4.0 International. 


\section{YAROSLAVA GENOVA Université de Plovdiv}

\section{LA CONSTITUTIONNALITÉ DE LA RÉFORME DE LA SÉCURITÉ SOCIALE}

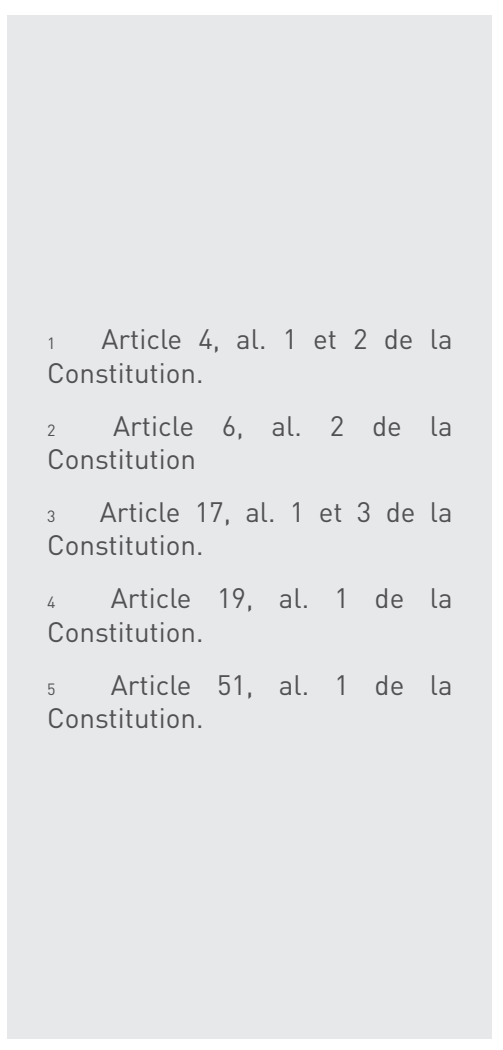

Par Décision n9 du 6 juillet 2017, la Cour Constitutionnelle a confirmé la constitutionnalité de l'article 4b, al. 1, de l'article 4c, alinéa 1 et de l'article 124a, al.1 du Code de la sécurité sociale. Il est nécessaire de rappeler ici que ces trois articles étaient très controversés du fait d'avoir introduit des aspects importants et radicalement novateurs aux termes de la réforme des pensions adoptée début de 2016. Il s'agissait, au fond, d'un détournement de la philosophie même du système de la sécurité sociale tel qu'il avait été édifié avec sa codification en 2000. En effet ces règles nouvellement introduites manipulaient les rapports entre les deux piliers obligatoires du système des pensions - le pilier solidaire et le pilier complémentaire par capitalisation. C'est pourquoi, le $1^{\text {er }}$ juin 2016 , des juges de la Cour suprême administrative ont utilisé leur faculté d'engager une procédure de contrôle de constitutionnalité auprès de la Cour constitutionnelle. Les enjeux -à la fois juridique, politique, financier et social, de l'affaire étaient de taille. Ce n'est, donc, pas étonnant que les juges constitutionnels se prononcent après plus d'un an de réflexions et en plus par la négative. Il leur revenait de vérifier la conformité desdites normes du Code de la sécurité sociale au principe constitutionnel de l'État de droit et de sauvegarde des droits et de la dignité des citoyens ${ }^{1}$, au principe d'égalité de traitement et de non discrimination ${ }^{2}$, au droit de propriété privée ${ }^{3}$, à la liberté économique des entrepreneurs ${ }^{4}$ et au droit de la sécurité sociale ${ }^{5}$.

L'article 4b, al. 1 du Code de la sécurité sociale concerne les personnes assurées nées après le 31 décembre 1959 qui étaient, jusqu'à son entrée en vigueur, assujetties obligatoirement à cotiser à la fois au Fonds «Pensions » de l'Institut national des assurances (premier pilier du système des pensions), donnant droit à la pension de retraite de base, et à un des fonds de pensions universels, gérés par des sociétés actionnaires d'assurance de retraite (second pilier par capitalisation), ouvrant droit à une pension complémentaire. La nouvelle règle permet de rompre avec le fonds privé en transférant la cotisation de $5 \%$ pour la pension complémentaire vers le Fonds «Pensions » et ainsi de faire le choix de participer seulement au premier pilier, mais avec la cotisation de base $(13,8 \%)$ à laquelle s'ajoute la cotisation complémentaire 
(5\%). Cela ouvrirait droit à une seule pension de retraite, mais avec une majoration résultant de l'augmentation de la cotisation. Le choix de l'assuré, réversible sans limitation grâce à la règle de l'article 124a, al. 1 du Code de la sécurité sociale, ne devient pérenne que cinq ans avant l'âge de la retraite. L'article 4c, al.1 du Code prévoit pareille possibilité de « passerelle » pour les cotisations complémentaires vers le Fonds solidaire « Pensions » en faveur des assurés travaillant dans des conditions difficiles, obligatoirement assurés pour une pension temporaire de retraite professionnelle anticipée près d'un fonds privé par capitalisation dits « professionnels », le choix étant pour ceci définitif.

Outre les juges de la Cour Suprême administrative qui ont saisi la Cour Constitutionnelle, l'avis de non-conformité constitutionnelle desdites règles était partagé par L'Association bulgare des sociétés d'assurance complémentaire de retraite, par une des grandes organisations patronales et par le doyen de la doctrine du droit de la sécurité sociale lle professeur Mratchkov). L'avis contraire était soutenu par les représentants de l'État, par l'Agence nationale des revenus, par l'Institut national assurantiel en tant qu'organisme gérant le Fonds « Pensions », par une organisation patronale représentative et, ce qui est plus curieux, par toutes les organisations syndicales représentatives ainsi que par un autre représentant de la doctrine (le professeur Sredkova).

Concernant la décision, la première chose qui frappe c'est qu'il s'agit d'un texte assez court avec des motifs laconiques. Si peu de mots pour n'être pas malheureusement convaincant. Au contraire, on soupçonne que des raisons fiscales et politiques se soient imposées aux principes constitutionnels du droit de la sécurité sociale. En outre, bien que les juges constitutionnels prétendent avoir trouvé leurs arguments dans une pratique constante de la Cour Constitutionnelle en matière de sécurité sociale (trois décisions précédentes sont en effet mentionnées, dans lesquelles on n'a puisé que l'argument jugé opportun, extrait de son propre contexte et ignorant tous les autres raisonnements et interprétations également applicables en l'espèce), ils rompent en réalité avec le sens que ces décisions donnaient au droit fondamental des citoyens à la sécurité sociale, proclamé par l'article 51 de la Constitution. Jusqu'à présent la doctrine bulgare du droit de la sécurité sociale, ainsi que le juge constitutionnel dans de nombreuses décisions n'ont jamais contesté que l'assurance complémentaire obligatoire de retraite a le caractère d'une assurance sociale et, en tant que telle forme, le second pilier du système des pensions. Ce type d'assurance, bien que fondé sur le principe de la capitalisation des cotisations et géré par des sociétés par actions, a, depuis sa création en 2000 , été regardé comme faisant partie du système de la sécurité sociale. C'était évidemment aussi le dessein du législateur vue la structure du Code de la sécurité sociale dans lequel l'assurance complémentaire obligatoire de retraire est traitée après le régime de base dit « Sécurité sociale étatique ». L'emblématique décision de la Cour Constitutionnelle $n^{\circ} 5$ de 2000 a confirmé sans équivoque la conformité de ce second pilier du système des pensions, à l'époque tout juste introduit par l'article 51, al. 1 de la Constitution. Le juge constitutionnel avait alors souligné que grâce à ce nouveau second pilier, basé sur la capitalisation personnalisée des cotisations, la Bulgarie 
comme État social réalisait pleinement et garantissait le droit des citoyens à la sécurité sociale dont le droit à la pension de retraite faisait partie. Cela explique la préoccupation créée par les nouvelles règles dites de «passerelle » entre les deux piliers du système des pensions.

Pour démontrer la conformité des règles contestées au droit à la sécurité sociale, les juges constitutionnels ont effectué une interprétation littérale douteuse et artificielle aux fins d'expliquer le sens du terme « sécurité sociale ». À leur avis la sécurité provient de l'action de procurer, de garantir, de donner, et d'assurer. Et la sécurité est sociale quand la société en tant que telle est impliquée dans l'action d'assurer l'assuré qui en tant que membre de la société a le droit de profiter de la « sécurité », mais a aussi l'obligation de contribuer à l'assurance des autres membres. D'où le fait que la sécurité sociale est synonyme d'assurance mutuelle et ne peut donc être que solidaire. Inversement, ce qui n'est pas solidaire, ne relève pas de la sécurité sociale. Il résulte, de cette interprétation délibérément restrictive, que, paradoxalement, le second pilier du système des pensions en Bulgarie ne fait pas partie de la sécurité sociale car il n'est pas conçu sur le principe de la solidarité mais basé sur celui de la capitalisation individualisée des cotisations. C'est pourquoi, le droit subjectif d'être assuré par un fonds géré par une société par actions d'assurance de retraite complémentaire n'est pas du tout un droit social mais un droit civil relevant du droit des obligations. Tout cela a permis à la Cour Constitutionnelle d'en conclure que toute modification législative du régime juridique de l'assurance complémentaire obligatoire de retraite, n'a rien à voir avec le droit constitutionnel à la sécurité sociale et partant ne peut en aucun cas l'enfreindre.

L'autre préoccupation majeure provoquée par les nouvelles règles résidait dans les insécurités que le droit d'option reconnu par l'article 4b, a. 1 et 124a, alinéa 1 du Code de la sécurité sociale, créait pour le citoyen. L'impossibilité pour le particulier d'estimer à l'avance l'impact de sa décision de cesser de s'assurer auprès du second pilier sur le montant de sa future pension, rend ce choix complètement hasardeux. La liberté d'agir n'est pas une liberté réelle si on n'agît pas en connaissance de cause. C'est pourquoi les juges de la Cour Suprême administrative ont estimé qu'il y avait infraction au principe de la sécurité juridique intrinsèque à tout État de droit. Ce à quoi la Cour Constitutionnelle a rétorqué : « ignorantia juris neminem excusat», soulignant que l'assuré peut et doit s'informer des effets juridiques directs de ses choix. Concernant les effets économiques à long terme, les juges constitutionnels ont rejeté chaque 
considération vue comme paternaliste et incompatible avec le principe de la liberté de la personne. L'assuré, agissant comme citoyen libre à ses risques et périls, doit assumer aussi le risque économique inhérent à son choix de quitter le second pilier du système des pensions et d'opter pour seulement le premier pilier. Curieusement, les juges constitutionnels voient dans ce choix une nouvelle facette du principe constitutionnel du principe de la libre initiative économique (article 19, al. 1 de la Constitution). Ils insistent sur le fait que les assurés dans les fonds par capitalisation sont ainsi impliqués dans une activité marchande, dans le commerce, tout en ignorant la différence existant entre les associés des sociétés d'assurance complémentaire de retraite pour lesquels l'assurance est une forme d'investissement, et les assurés pour lesquels l'assurance est créatrice d'un statut en vu de l'acquisition des droits à pension. Mais pour la Cour Constitutionnelle, si l'assuré décide de quitter le fonds par capitalisation en transférant les cotisations complémentaires vers le Fonds solidaire «Pensions », cela relève de sa liberté de " sortir du commerce ». Avec audace et laconisme, les juges constitutionnels ont rejeté de la même manière l'argument selon lequel les nouvelles règles de «passerelle » provoquent une situation d'inégalité entre assurés. Enfin, la Cour se contente de considérer simplement que les nouvelles normes s'adressant à tous les assurés ne sont pas contraires à l'article 6, al. 1 de la Constitution consacrant le principe de l'égalité devant la loi.

La dernière préoccupation des juges de la Cour Suprême administrative était que les règles contestées avaient pour effet secondaire de diminuer potentiellement le nombre de personnes assurées dans le second pilier. Le transfert des cotisations complémentaires pourrait mettre à l'épreuve les fonds de pensions complémentaires par capitalisation, tout en favorisant la stabilité et la crédibilité du Fonds « Pensions » bénéficiant de financement supplémentaire du fait du choix des assurés. Théoriquement cela pourrait déclencher un processus qui obligerait en fin de compte certaines sociétés par actions d'assurance complémentaire retraite de sortir définitivement du marché assurantiel en cessant de gérer ce type de fonds. Mais la Cour Constitutionnelle a dénié l'atteinte au principe constitutionnel (article 19, al. 2 de la Constitution) de l'égalité des conditions d'exercice de l'activité économique en préservant des monopoles et de la concurrence déloyale les personnes morales. L'argumentation formelle se bornait à pointer que l'Institut national assurantiel gère le Fonds «Pensions » qui fait partie de la sécurité sociale et que ledit Institut n'a pas d'activité économique et n'est donc pas un concurrent des sociétés d'assurances complémentaires. Et, quand bien même, le nombre décroissant de personnes assurées dans les fonds par capitalisation aiguiserait la concurrence entre les sociétés gérantes de ces fonds, cela ne serait pas constitutif de concurrence déloyale. 